\title{
Resisting and tolerating $P$. falciparum in pregnancy under different malaria transmission intensities
}

\author{
Nicaise Tuikue Ndam ${ }^{1,2,3}$, Emmanuel Mbuba ${ }^{4}$, Raquel González ${ }^{5,6}$, Pau Cisteró ${ }^{5}$, Simon Kariuki ${ }^{8}$, \\ Esperança Sevene ${ }^{6,7}$, María Rupérez ${ }^{5,6}$, Ana Maria Fonseca ${ }^{5,9}$, Anifa Vala ${ }^{6}$, Sonia Maculuve ${ }^{6}$, Alfons Jiménez ${ }^{5,10}$, \\ Llorenç Quintó $^{3}$, Peter Ouma ${ }^{8}$, Michael Ramharter ${ }^{11,12,13}$, John J. Aponte ${ }^{5,6}$, Arsenio Nhacolo ${ }^{6}$, \\ Achille Massougbodji ${ }^{3}$, Valerie Briand ${ }^{1}$, Peter G. Kremsner ${ }^{11,13}$, Ghyslain Mombo-Ngoma ${ }^{11,13}$, Meghna Desai ${ }^{14}$, \\ Eusebio Macete ${ }^{6}$, Michel Cot $^{1}$, Clara Menéndez ${ }^{5,6+}$ and Alfredo Mayor ${ }^{5,6^{*}+}$
}

\begin{abstract}
Background: Resistance and tolerance to Plasmodium falciparum can determine the progression of malaria disease. However, quantitative evidence of tolerance is still limited. We investigated variations in the adverse impact of $P$. falciparum infections among African pregnant women under different intensities of malaria transmission.

Methods: $P$. falciparum at delivery was assessed by microscopy, quantitative PCR (qPCR) and placental histology in 946 HIV-uninfected and 768 HIV-infected pregnant women from Benin, Gabon, Kenya and Mozambique. Resistance was defined by the proportion of submicroscopic infections and the levels of anti-parasite antibodies quantified by Luminex, and tolerance by the relationship of pregnancy outcomes with parasite densities at delivery.

Results: P. falciparum prevalence by GPCR in peripheral and/or placental blood of HIV-uninfected Mozambican, Gabonese and Beninese women at delivery was 6\% (21/340), 11\% (28/257) and 41\% (143/349), respectively. The proportion of peripheral submicroscopic infections was higher in Benin (83\%) than in Mozambique (60\%) and Gabon (55\%; $P=0.033)$. Past or chronic placental $P$. falciparum infection was associated with an increased risk of preterm birth in Mozambican newborns ( $\mathrm{OR}=7.05,95 \% \mathrm{Cl} 1.79$ to 27.82). Microscopic infections were associated with reductions in haemoglobin levels at delivery among Mozambican women $(-1.17 \mathrm{~g} / \mathrm{dL}, 95 \% \mathrm{Cl}-2.09$ to -0.24$)$ as well as with larger drops in haemoglobin levels from recruitment to delivery in Mozambican $(-1.66 \mathrm{~g} / \mathrm{dL}, 95 \% \mathrm{Cl}$ -2.68 to -0.64$)$ and Gabonese $(-0.91 \mathrm{~g} / \mathrm{dL}, 95 \% \mathrm{Cl}-1.79$ to -0.02$)$ women. Doubling qPCR-peripheral parasite densities in Mozambican women were associated with decreases in haemoglobin levels at delivery $(-0.16 \mathrm{~g} / \mathrm{dL}$, $95 \% \mathrm{Cl}-0.29$ to -0.02$)$ and increases in the drop of haemoglobin levels $(-0.29 \mathrm{~g} / \mathrm{dL}, 95 \% \mathrm{Cl}-0.44$ to -0.14$)$. Beninese women had higher anti-parasite IgGs than Mozambican women $(P<0.001)$. No difference was found in the proportion of submicroscopic infections nor in the adverse impact of $P$. falciparum infections in HIV-infected women from Kenya (P. falciparum prevalence by qPCR: 9\%, 32/351) and Mozambique (4\%, 15/417).

(Continued on next page)
\end{abstract}

\footnotetext{
* Correspondence: alfredo.mayor@isglobal.org

${ }^{\dagger}$ Equal contributors

${ }^{5}$ ISGlobal, Barcelona Ctr. Int. Health Res. (CRESIB), Hospital Clínic - Universitat de Barcelona, Barcelona, Spain

${ }^{6}$ Centro de Investigação em Saúde da Manhiça (CISM), Maputo, Mozambique

Full list of author information is available at the end of the article
} 
(Continued from previous page)

Conclusions: The lowest levels of resistance and tolerance in pregnant women from areas of low malaria transmission were accompanied by the largest adverse impact of $P$. falciparum infections. Exposure-dependent mechanisms developed by pregnant women to resist the infection and minimise pathology can reduce malaria-related adverse outcomes. Distinguishing both types of defences is important to understand how reductions in transmission can affect malaria disease.

Trial registration: ClinicalTrials.gov NCT00811421. Registered 18 December 2008.

Keywords: Malaria, Pregnancy, Immunity, Resistance, Tolerance

\section{Background}

As the rest of the population, pregnant women living in areas of different malaria endemicity experience varying degrees of exposure to infection, which affects the acquisition of antimalarial immunity and determines the course of disease $[1,2]$. Protective immunity to Plasmodium falciparum in pregnancy has been suggested to rely mostly on antibodies against VAR2CSA that block adhesion of infected erythrocytes to placental chondroitin sulphate A, and thereby prevent parasite sequestration in the placenta [3]. Such immune resistance to P. falciparum, which is acquired after exposure to placental-type parasites, reduces parasite densities below the detection limit of microscopy (i.e. submicroscopic infections) [4-6] and can eventually clear placental infections.

Infected hosts may also tolerate the presence of $P$. falciparum by minimising parasite-induced damage without necessarily limiting the infection [7-9]. This type of host defence, not to be confused with immunological tolerance $[7,10]$, has been suggested by the frequent observation in malaria endemic areas of individuals, including pregnant women, who harbour levels of parasitaemia in their blood that would commonly be associated with fever in malaria-naïve individuals [11, 12]. Moreover, the higher risk of life-threatening disease in younger age groups [13] supports the notion that the ability to modulate host inflammation (anti-disease or clinical immunity) [14, 15] develops faster than the capacity to restrict parasite growth (anti-parasite immunity). However, other studies do not support the hypothesis that a special clinical immunity exists independently of parasitological immunity [16], but rather suggest that immunity resulting in decreased parasite densities reduces the severity of symptoms.

Resolving the role of resistance and tolerance could aid the development of host-directed therapies to reduce malaria-induced immunopathology and mitigate malaria disease [9]. However, quantitative analyses of tolerance to human malaria have been mainly limited to the assessment of peripheral $P$. falciparum parasitemia needed to trigger the onset of fever (i.e. pyrogenic threshold) $[14,15]$. Alternative clinical outcomes and analytical frameworks are needed for pregnant women in whom parasitaemia is poorly associated with fever [17]. Here, we aimed to assess the variations in the clinical impact of $P$. falciparum infections and in host defences developed by pregnant women under different malaria transmission intensities. To achieve this, we compared the carriage of submicroscopic infections and antibodies against $P$. falciparum antigens as indicators of the level of parasitological immunity [1]. We assessed the correlation between health outcomes (haemoglobin levels and birthweight) and parasite densities at delivery for summarising tolerance [8], with a flat slope indicative of tolerance to infection. As immune resistance is strongly influenced by the number of previous pregnancies in areas of stable transmission [18] and pregnancy outcomes can be affected by the preventive measures used during pregnancy, analyses were adjusted for parity of the pregnant women and the antimalarials received as intermittent preventive treatment during pregnancy (IPTp).

\section{Methods}

\section{Study populations}

This study was conducted between 2010 and 2012 in four sub-Saharan countries (Additional file 1: Figure S1), namely Benin (Allada, Sékou and Attogon), Gabon (Lambaréné and Fougamou), Kenya (Siaya), and Mozambique (Manhiça and Maragra). Pregnant women were enrolled in the context of the Malaria in Pregnancy Preventive Alternative Drugs clinical trial (ClinicalTrials.gov NCT0081121; Table 1) [19, 20]. At enrolment, pregnant women received long-lasting insecticide-treated bed-nets. Following national guidelines in place, HIV status was assessed after voluntary HIV counselling and testing with an HIV rapid test and positive results were confirmed with a second rapid test $[19,20]$. Haemoglobin and the syphilis rapid plasma reagin (RPR) test were assessed as part of routine antenatal care on finger-prick-collected capillary blood. Among HIV-infected women, $5 \mathrm{~mL}$ of venous blood were taken for $\mathrm{CD} 4+\mathrm{T}$ cell count by flow cytometry after staining of whole blood with CD3, CD8 and CD4 fluorochrome-labelled antibodies. Flow cytometry acquisition was performed using FACSCalibur (BD Biosciences) and TruCOUNT tubes (Becton Dickinson). Additionally, viral load determination was performed using the COBAS 
Table 1 Characteristics of participants by study area

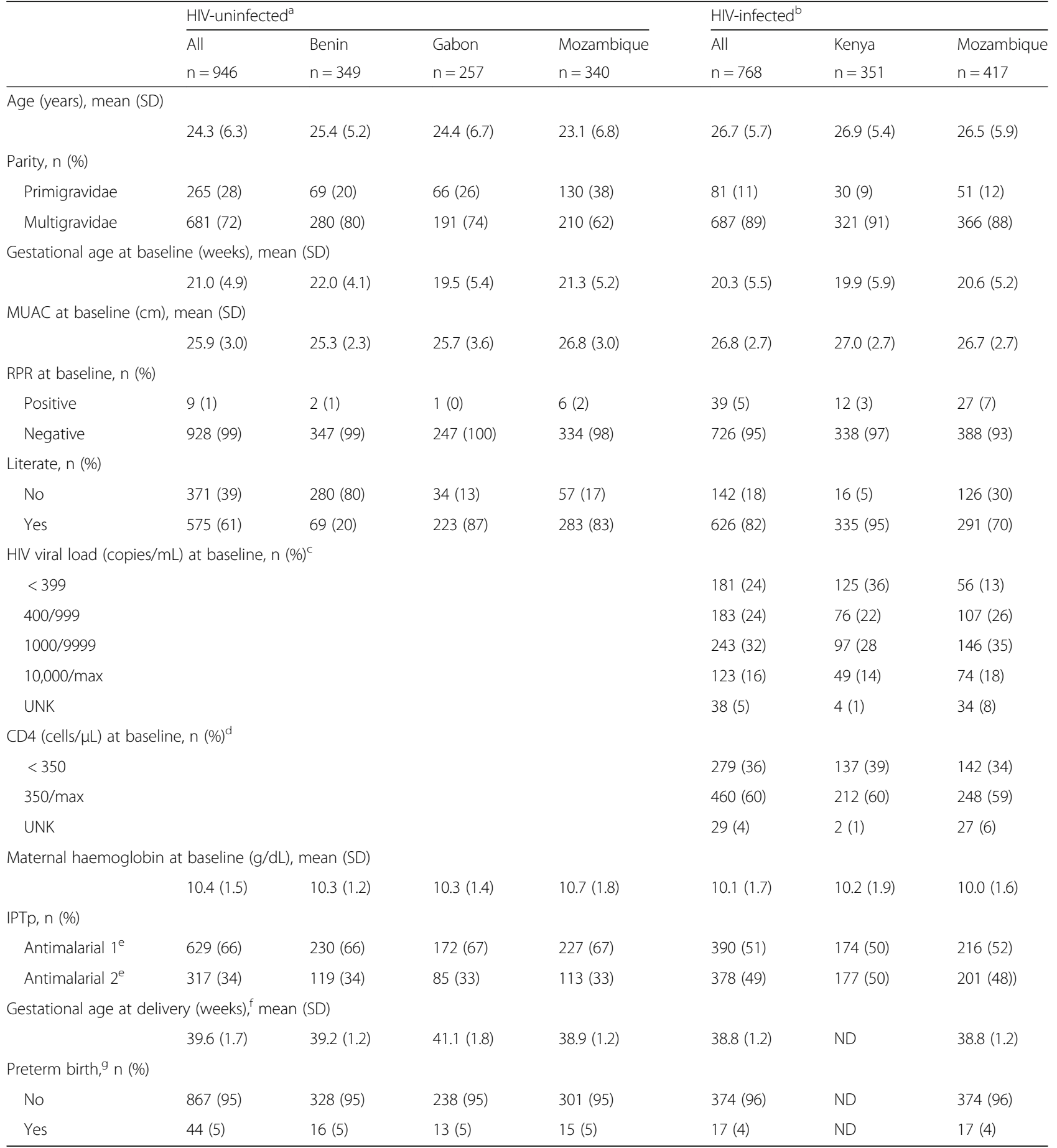

${ }^{a}$ The IPTp trial evaluated the efficacy and safety of two doses of Intermittent Preventive Treatment in pregnancy (IPTp) with mefloquine compared to IPTp with sulphadoxine-pyrimethamine

${ }^{\mathrm{b}}$ Evaluated three doses of IPTp with MQ compared to placebo in HIV-infected women on cotrimoxazole prophylaxis

c 38 missing values

d 29 missing values

eAntimalarial: 1 = Mefloquine and 2 = Sulphadoxine-Pyrimethamine for HIV-uninfected; 1 = Placebo and 2 = Mefloquine for HIV-infected

fGestational age was estimated by newborn physical examination using the Ballard score

gPreterm birth if gestational age was $<37$ weeks

IPTp intermittent preventive treatment, MUAC mid-upper arm circumference, RPR rapid plasma reagin, UNK unknown 
AMPLICOR or AmpliPrep (Roche Diagnostics) devices. Haemoglobin was determined in capillary blood samples using mobile devices (HemoCue and Hemocontrol) [19, 20]. Gestational age at enrolment was determined from symphysis fundal height measurement using a standard tape measure (centimetres) and McDonald's rule to transform the symphysis fundal height in centimetres into gestational weeks $[19,20]$. Following physical examination, recruited women with gestational age $\geq 13$ weeks received their first dose of IPTp (either sulfadoxine-pyrimethamine (SP) or mefloquine (MQ) for HIV-uninfected women and either placebo or MQ for HIV-infected women) under supervision. Women allocated to the SP group received standard IPTp (three tablets of the fixed combination therapy containing $500 \mathrm{mg}$ of sulfadoxine and $25 \mathrm{mg}$ of pyrimethamine), whereas participants allocated to the MQ groups received $15 \mathrm{mg} / \mathrm{kg}$ of the drug. The second IPTpSP/MQ administration for HIV-uninfected women, and the second and third administrations of IPTp-MQ/ placebo for HIV-infected women, were given at least 1 month apart. All HIV-infected women also received study co-trimoxazole tablets on a monthly basis for daily prophylaxis.

At delivery, maternal haemoglobin was determined and newborns were weighed using weekly calibrated scales (either digital or three beam balances) and their gestational age at birth (except for Kenyan newborns) was evaluated using the Ballard's score [21]. Newborn weights not captured at birth but within the first week of life were estimated using a linear regression model [22]. Peripheral and placental blood smears, as well as $50 \mu \mathrm{L}$ of maternal peripheral and placental blood spotted onto filter paper, were collected for parasitological assessments. Tissue samples were also collected from the maternal side of the placenta and placed into $10 \%$ neutral buffered formalin. Biopsies were processed, stained and examined following standard procedures [23]. From the first antenatal visit, all study women were indicated to receive ferrous sulphate-folic acid supplements for prevention of anaemia in pregnancy. If a woman was diagnosed with anaemia, she was treated with oral ferrous sulphate $200 \mathrm{mg} / 8$ hours for 3 months or blood transfusion for severe cases. Clinical malaria episodes were treated with oral quinine (first trimester) or artemetherlumefantrine (subsequent trimesters) for uncomplicated malaria; parenteral quinine was used for treatment of severe malaria $[19,20]$.

\section{Parasitological determinations}

Thick and thin blood films, as well as placental biopsies, were read for Plasmodium species detection according to standard, quality-controlled procedures [19, 20, 24]. The quality of the reading across sites was controlled through a quality control program during the study. Past placental infection was defined by the presence of $P$. falciparum pigment (i.e. hemozoin) without parasite detection on placental histologic examination, and chronic placental infection was defined by the presence of $P$. falciparum pigment in combination with the detection of parasites [1]. A 30\% random selection of paired peripheral and placental blood onto filter papers from HIVnegative women [20] and all the paired filter papers from HIV-infected women [19] were tested for the presence and density of $P$. falciparum in duplicate by means of a real-time quantitative polymerase chain-reaction (qPCR) targeting 18S rDNA [1, 25]. Parasitemia was quantified by extrapolation of cycle thresholds from a standard curve of $P$. falciparum ring-infected erythrocytes. Samples without amplification (no cycle thresholds detected) were considered negative, and a density of 2 parasites $/ \mu \mathrm{L}$ was assigned if amplification was observed out of the lower range of the standard curve (5 parasites $/ \mu \mathrm{L}$ ). A negative control with no template DNA was run in all reactions. P. falciparum infections were considered submicroscopic if parasites were detected by qPCR but not by microscopy [1]. A quality check program was established to ensure comparable performance of qPCR techniques in different laboratories [25].

\section{Measurement of antimalarial IgGs and $P$. falciparum Histidine-Rich Protein 2 (PfHRP2)}

A random selection of $50 \%$ of peripheral plasma samples collected at delivery from HIV-uninfected women in the extremes of the malaria transmission spectrum $(n=170$ from Benin and $n=170$ from Mozambique) was tested for IgG levels against $P$. falciparum recombinant VAR2CSA domain (DBL3X from 3D7 strain), and the merozoite surface protein-1 (MSP-1 $19 ; 19-\mathrm{kD}$ fragment, 3D7 strain) using a multiplex suspension array panel $\left(\mathrm{xMAP}^{\mathrm{TM}}\right.$ technology) and the Luminex ${ }^{\oplus} 100 / 200^{\text {TM }}$ System (Luminex Corp., Austin, TX, USA) [1]. Briefly, magnetic carboxylated microspheres (MagPlex ${ }^{\text {TM }}-\mathrm{C}$, Luminex) were coupled with recombinant protein. After blocking with bovine serum albumin in phosphate-buffered saline, microspheres were sequentially incubated with $100 \mu \mathrm{L}$ of plasma (dilutions 1:500, 1:20,000 and 1:800,000 in duplicate for each sample), $100 \mu \mathrm{L}$ of biotinylated anti-human IgG (diluted 1:2500) and $100 \mu \mathrm{L}$ of streptavidinconjugated R-phycoerythrin (diluted 1:1000). The plate was immediately read using Bio-Plex Manager version 4.0, and at least 50 microspheres per analyte were acquired per sample. Crude mean fluorescent intensity was exported with background fluorescence from blank wells already subtracted. PfHRP2 [26] was quantified in plasmas available from women with peripheral qPCR-detected $P$. falciparum infection at delivery $(n=42$ in Benin and $n=$ 13 in Mozambique) using a commercial HRP2 antigencapture ELISA (Malaria Ag CELISA kit; Cellabs) [27]. 


\section{Statistical analysis}

Pregnant women were included in the analysis if they had all information on IPTp type of treatment, HIV infection, age, parity, newborn weight, maternal haemoglobin levels, as well as qPCR results in peripheral and placental blood at time of delivery. Women were classified as primigravid (first pregnancy) and multigravid (at least one previous pregnancy). Age was categorised as $<25$ and $\geq 25$ years on the basis of median maternal age in the study population. Preterm birth was defined as a gestational age $<37$ weeks. Participant's baseline characteristics, parasitological outcomes, antimalarial IgG levels and PfHRP2 levels were compared between study areas by univariate analysis and logistic or lineal regression models. Changes in maternal haemoglobin levels and birthweight with increases in qPCR-peripheral parasite density were assessed by linear regression. The impact of $P$. falciparum infections on haemoglobin levels, birthweight and preterm birth was assessed in logistic regression models. Continuous outcome variables (qPCR-parasite densities, antimalarial IgGs and HRP2 levels) exhibiting a skewed distribution were log transformed. Regression models were estimated through a backward stepwise approach with a significance level for removal from the model of 0.1 and a significance level for addition of 0.05 , adjusted by type of IPTp drug and baseline covariates at recruitment (season, age, gravidity, gestational age, anaemia, literacy, RPR result and midupper arm circumference (MUAC)), as well as CD4 + T cell count in the case of HIV-infected women [19, 20]. The modification of the associations by study area was assessed by including interaction terms into the regression models and combining the coefficients plus the interaction and the standard error by the delta method. $P$ values of less than 0.05 were considered to indicate statistical significance. Stata version 13 was used for data analysis and GraphPad version 5.01 for graphical depiction of data.

\section{Results}

\section{$P$. falciparum burden in the study populations}

A total of 1714 pregnant women, 946 (55\%) HIVuninfected and 768 (45\%) HIV-infected, were included in this study (Table 1 and Additional file 1: Figure S1). The 3428 peripheral and placental filter papers from these $946 \mathrm{HIV}$-uninfected and $768 \mathrm{HIV}$-infected women were tested for the presence and density of $P$. falciparum by qPCR $[25,28]$. Covariates at recruitment were similar in the study subgroup and the rest of the women participating in the randomised trial $[19,20]$ (Additional file 1: Table S1). Prevalence of qPCR-detected P. falciparum at delivery, either in peripheral blood at delivery or placental blood (maternal qPCR infection in Table 2), was $41 \%$ $(143 / 349)$ in Benin, 11\% (28/257) in Gabon and 6\% (21/ 340) in Mozambique $(P<0.001)$ among HIV-uninfected women, and 9\% (32/351) in Kenya and 4\% (15/417) in Mozambique $(P<0.001)$ among HIV-infected women (Fig. 1a and Table 2). Similar trends were observed for maternal microscopic infections (Fig. 1b and Table 2). The relationship between microscopy positivity and qPCR parasitaemia in the different study sites is presented in Additional file 1 (Table S2). The proportion of samples that were negative by microscopy but for which qPCR indicated a parasitaemia above 200 parasites $/ \mu \mathrm{L}$ was not different between sites (Additional file 1: Table S3).

\section{Effects of $P$. falciparum infection on health outcomes}

Microscopic $P$. falciparum infection detected at delivery in peripheral blood and/or the placenta of Mozambican women was associated with a reduction of $1.17 \mathrm{~g} / \mathrm{dL}(95 \%$ CI -2.09 to $-0.24, P=0.014$ ) in their haemoglobin levels, but this reduction was not observed among Beninese nor Gabonese women $(P=0.581$ and $P=0.154$, respectively; Fig. 2a). Microscopic infections were also associated with larger drops in haemoglobin levels from recruitment to delivery in Mozambican $(-1.66 \mathrm{~g} / \mathrm{dL}, 95 \% \mathrm{CI}-2.68$ to $0.64, P=0.001)$ and Gabonese $(-0.91 \mathrm{~g} / \mathrm{dL}, 95 \% \mathrm{CI}-1.79$ to $-0.02, P=0.045)$ women, but not among Beninese women $(P=0.213$; Fig. $2 \mathrm{c})$. No impact of microscopic $P$. falciparum infection in the mother was observed on the birthweight, irrespective of study area (Fig. 2e). However, past or chronic placental P. falciparum infection, defined by the presence of $P$. falciparum pigment (i.e. hemozoin) on placental histologic examination, was associated with a reduction of $247.2 \mathrm{~g}$ (95\% CI -479.1 to $-15.2, P=0.037)$ in the birthweight of Mozambican babies, which was not observed in Benin $(P=0.569)$ and Gabon $(P=0.486)$. Given the strong association between birthweight and gestational age at delivery (Additional file 1: Table S4), the regression model assessing the relationship between birthweight and infection was adjusted by preterm birth, showing a loss of statistical significance $(P=0.192)$. However, past or chronic placental $P$. falciparum infection were associated with an increased risk of preterm birth in Mozambican newborns $(\mathrm{OR}=7.05,95 \% \mathrm{CI} 1.79$ to $27.82, P=$ $0.005)$, but not among Beninese $(P=0.785)$ and Gabonese newborns $(P=0.639)$. Microscopic $P$. falciparum infection was not associated with reductions in haemoglobin levels of HIV-infected women from Kenya and Mozambique nor with reductions of birthweight (Fig. 2b, d, f). Finally, no adverse clinical impact was observed for $P$. falciparum submicroscopic infections, defined by the detection of $P$. falciparum by qPCR but not by microscopy, irrespective of the HIV-infectious status of the pregnant women (Additional file 1: Figure S2). Adjusting variables that remained in the final regression models and interactions assessed with parity, age and IPTp treatment are detailed in Table S5 and S6 of Additional file 1. 
Table 2 Parasitological outcomes in peripheral blood and placenta by study area

\begin{tabular}{|c|c|c|c|c|c|c|c|}
\hline & \multicolumn{4}{|l|}{ HIV-uninfected } & \multicolumn{3}{|l|}{ HIV-infected } \\
\hline & Benin & Gabon & Mozambique & $P$ & Kenya & Mozambique & $P$ \\
\hline \multicolumn{8}{|l|}{ Periphery at delivery } \\
\hline \multicolumn{8}{|l|}{ Smear, n (\%) } \\
\hline Negative & $320(92)$ & $245(96)$ & $333(98)$ & $0.002^{\mathrm{a}}$ & $332(95)$ & $408(98)$ & $0.025^{\mathrm{a}}$ \\
\hline Positive & $27(8)$ & $11(4)$ & $7(2)$ & & $17(5)$ & $8(2)$ & \\
\hline \multicolumn{8}{|l|}{ qPCR, n (\%) } \\
\hline Negative & $242(69)$ & $235(91)$ & $325(96)$ & $<0.001^{a}$ & $326(93)$ & $405(97)$ & $0.007^{\mathrm{a}}$ \\
\hline Positive & $107(31)$ & $22(9)$ & $15(4)$ & & $25(7)$ & $12(3)$ & \\
\hline 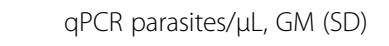 & $280.5(882.9)$ & $684.2(1703.2)$ & $514.4(1630.6)$ & 0.484 & $141.4(329.1)$ & $3350.9(9887.2)$ & 0.003 \\
\hline \multicolumn{8}{|l|}{ Placenta } \\
\hline \multicolumn{8}{|l|}{ Smear, n (\%) } \\
\hline Negative & $322(93)$ & $244(96)$ & $332(98)$ & $0.018^{\mathrm{a}}$ & $335(95)$ & $406(98)$ & $0.032^{\mathrm{a}}$ \\
\hline Positive & $23(7)$ & $9(4)$ & $8(2)$ & & $16(5)$ & $7(2)$ & \\
\hline \multicolumn{8}{|l|}{ Histology, n (\%) } \\
\hline Acute & $8(2)$ & $5(2)$ & $1(0)$ & $<0.001$ & $6(2)$ & $4(1)$ & $<0.001$ \\
\hline Chronic & $23(7)$ & $2(1)$ & $2(1)$ & & $5(1)$ & $1(0)$ & \\
\hline Past & $37(11)$ & $28(11)$ & $13(4)$ & & $64(18)$ & $14(3)$ & \\
\hline No infected & $277(80)$ & $218(86)$ & $322(95)$ & & $276(79)$ & $387(95)$ & \\
\hline \multicolumn{8}{|l|}{ qPCR, n (\%) } \\
\hline Negative & $253(72)$ & $235(91)$ & $323(95)$ & $<0.001^{a}$ & $324(92)$ & 407 (98) & $0.001^{a}$ \\
\hline Positive & $96(28)$ & $22(9)$ & $17(5)$ & & $27(8)$ & $10(2)$ & \\
\hline 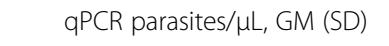 & $668.5(2473.1)$ & $1284.9(5100.0)$ & $1876.2(5910.5)$ & 0.609 & $379.5(1255.4)$ & $5851.3(19950.4)$ & 0.033 \\
\hline \multicolumn{8}{|l|}{ Maternal microcopy infection, ${ }^{b} \mathrm{n}(\%)$} \\
\hline Negative & $294(87)$ & $239(95)$ & $329(97)$ & $<0.001^{a}$ & $325(93)$ & $396(98)$ & $0.002^{\mathrm{a}}$ \\
\hline Positive & $45(13)$ & $12(5)$ & $9(3)$ & & $24(7)$ & $9(2)$ & \\
\hline \multicolumn{8}{|l|}{ Maternal qPCR infection, ${ }^{b}$ n (\%) } \\
\hline Negative & 206 (59) & 229 (89) & 319 (94) & $<0.001^{a}$ & 319 (91) & 402 (96) & $<0.001^{a}$ \\
\hline Positive & $143(41)$ & $28(11)$ & $21(6)$ & & $32(9)$ & $15(4)$ & \\
\hline
\end{tabular}

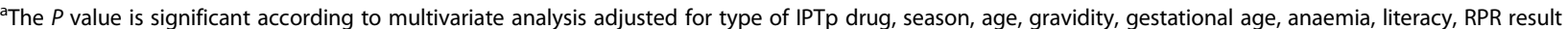
and MUAC at recruitment, as well as CD4 + T cell count in the case of HIV-positive women (Wald test) (ND for placental histology)

${ }^{b}$ Maternal infections were considered microscopic if $P$. falciparum parasites were observed in peripheral blood at delivery or in placental blood from the maternal side either by microscopy or histology and qPCR infections if peripheral blood at delivery or placental blood samples were positive by qPCR

GM geometric mean, SD standard deviation

\section{Submicroscopic $P$. falciparum infections, antimalarial immunity and HRP2 levels}

The proportion of peripheral submicroscopic infections among HIV-uninfected women was the highest in Benin (83\%; 82/106), followed by Mozambique $(60 \%, 9 / 15)$ and Gabon (55\%, 12/22, $P_{\text {adjusted }}=0.033$; Fig. 3a). Similar trends, although not statistically significant, were observed for placental submicroscopic infections (82\% (78/95), 58\% $(10 / 17)$ and $71 \%(15 / 21)$, respectively, $P_{\text {adjusted }}=0.674$; Fig. 3b). Among pregnant women with a qPCR-detected infection either in peripheral or placental blood, the proportion of submicroscopic infections in any of the two compartments was the highest in Benin (94\%, 124/132), followed by Gabon $(82 \%, 18 / 22)$ and Mozambique $(74 \%$, $\left.14 / 19, P_{\text {adjusted }}=0.028\right)$. PfHRP2 concentrations, indicative of overall parasite biomass [26], were higher in pregnant women with a peripheral qPCR-detected infection from Mozambique $(n=13)$ than from Benin $(n=42, P=0.048$; Fig. 3c). HRP2 levels decreased with parity of pregnant women in Benin $(0.13,95 \%$ CI 0.02 to $0.79, P=0.028)$ but not in Mozambique $(1.09,95 \%$ CI 0.09 to $12.21, P=$ $0.945)$. In contrast, age was not associated with differences in HRP2 levels among Beninese $(P=0.052)$ and Mozambican women $(P=0.123)$. Levels of IgGs against DBL3X from VAR2CSA and MSP1 were higher in women from Benin than in women from Mozambique $(P<0.007$; Fig. 3d). IgG responses against MSP1 increased with parity of Beninese pregnant women $(2.39,95 \%$ CI 1.49 to 

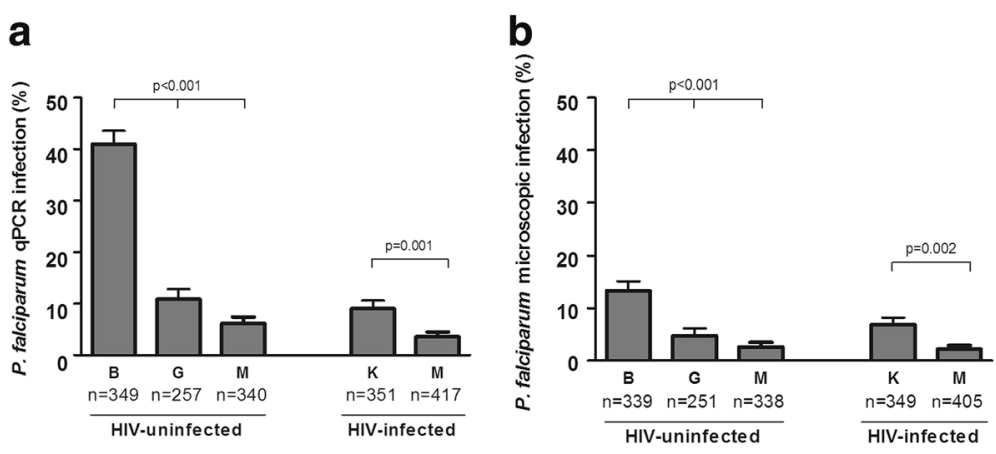

Fig. 1 Prevalence of $P$. falciparum maternal infection according to study area. Prevalence of $P$. falciparum at delivery in peripheral and/or placental blood as detected by qPCR (a) and microscopy (b). P value according to the multivariate analysis adjusted for type of IPTp drug, season, age, gravidity, gestational age, anaemia, literacy, RPR result and MUAC at recruitment, as well as CD4 + T cell count in the case of HIV-infected women. T bars represent standard errors. B Benin, G Gabon, M Mozambique, K Kenya

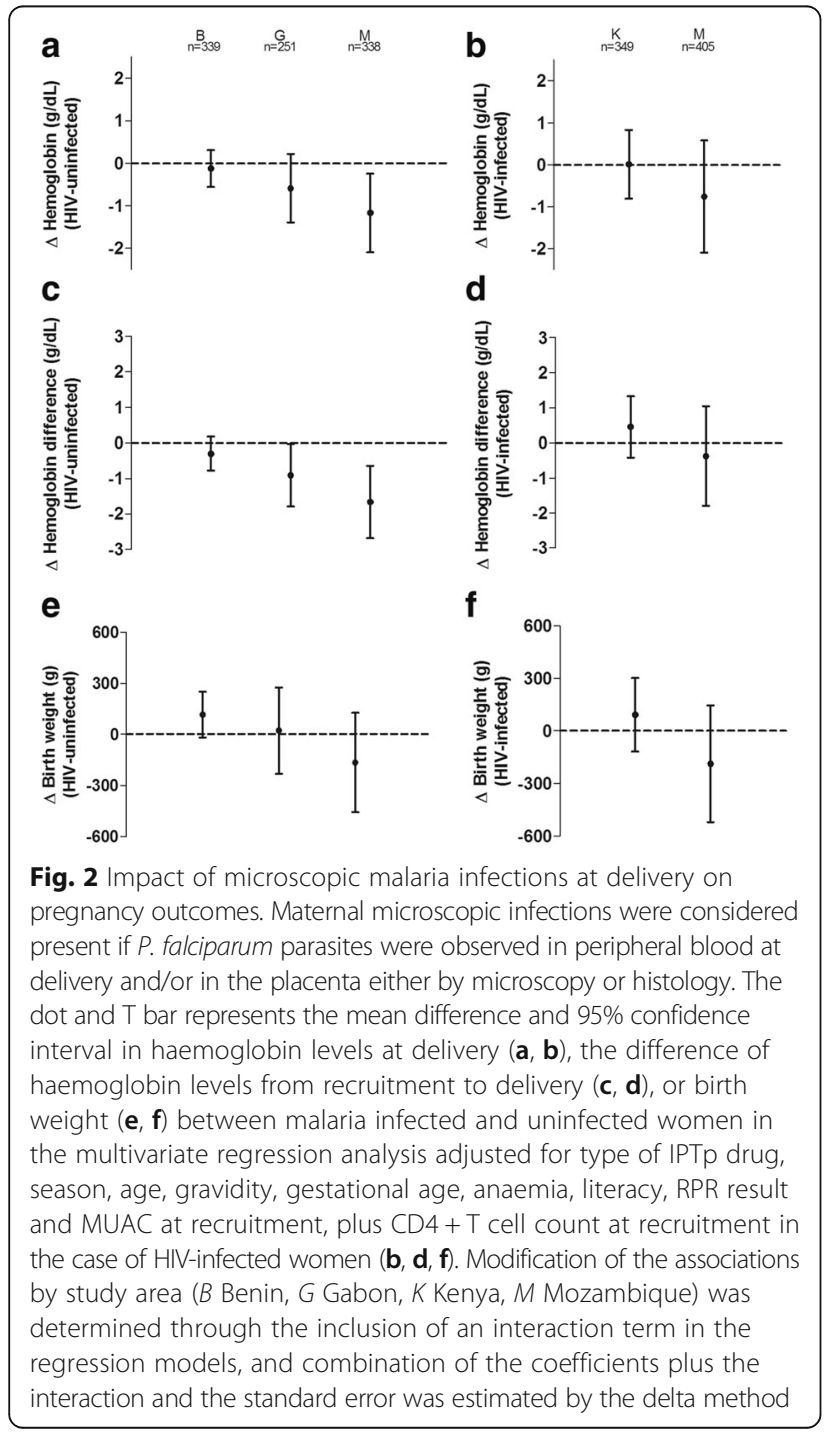

3.83, $P<0.001)$ but not among Mozambican pregnant women $(P=0.401)$. IgG responses against DBL3x increased with parity of Beninese and Mozambican pregnant women although the magnitude of the increase was higher among Beninese (4.77, 95\% CI 3.08 to $7.38, P<0.001)$ than Mozambican women (1.86, $95 \%$ CI 1.23 to $2.79, P=0.003$; Fig. 3e). Age was not associated with differences in IG responses irrespectively of antigen and study area $(P=0.223$ for MSP1 and $P=0.171$ for DBL3X in Benin and $P=0.959$ for MSP1 and $P=0.380$ for DBL3X in Mozambique). No differences in covariates at recruitment were observed between the women with plasma available for serological tests and the rest of the women. The proportion of submicroscopic infections did not differ among HIV-infected women from Kenya and Mozambique (Fig. 3a, b).

\section{$P$. falciparum densities and pregnancy outcomes}

Doubling qPCR parasite densities in peripheral blood of HIV-uninfected Mozambican women was associated with a reduction of $0.16 \mathrm{~g} / \mathrm{dL}(95 \% \mathrm{CI}-0.29$ to $-0.02, P$ $=0.023)$ in their haemoglobin levels. In contrast, haemoglobin levels remained unaffected by increasing parasite densities in Beninese $(P=0.287)$ and Gabonese women $(P=0.381$; Fig. 4a and Additional file 1: Figure S3). Similarly, doubling qPCR parasite densities in peripheral blood of HIV-uninfected Mozambican women was associated with a larger drop in the haemoglobin levels from recruitment to delivery $(-0.29 \mathrm{~g} / \mathrm{dL}, 95 \% \mathrm{CI}-0.44$ to $0.14, P<0.001)$, while no relationship was found in Beninese $(P=0.076)$ and Gabonese women $(P=0.176$; Fig. 4c and Additional file 1: Figure S3). No association was found between GPCR parasite densities and birthweight irrespectively of the country of women, nor with pregnancy outcomes in HIV-infected women (Fig. 4).

\section{Discussion}

Although the importance of tolerance in the context of $P$. falciparum infection was suggested early on [29], the 

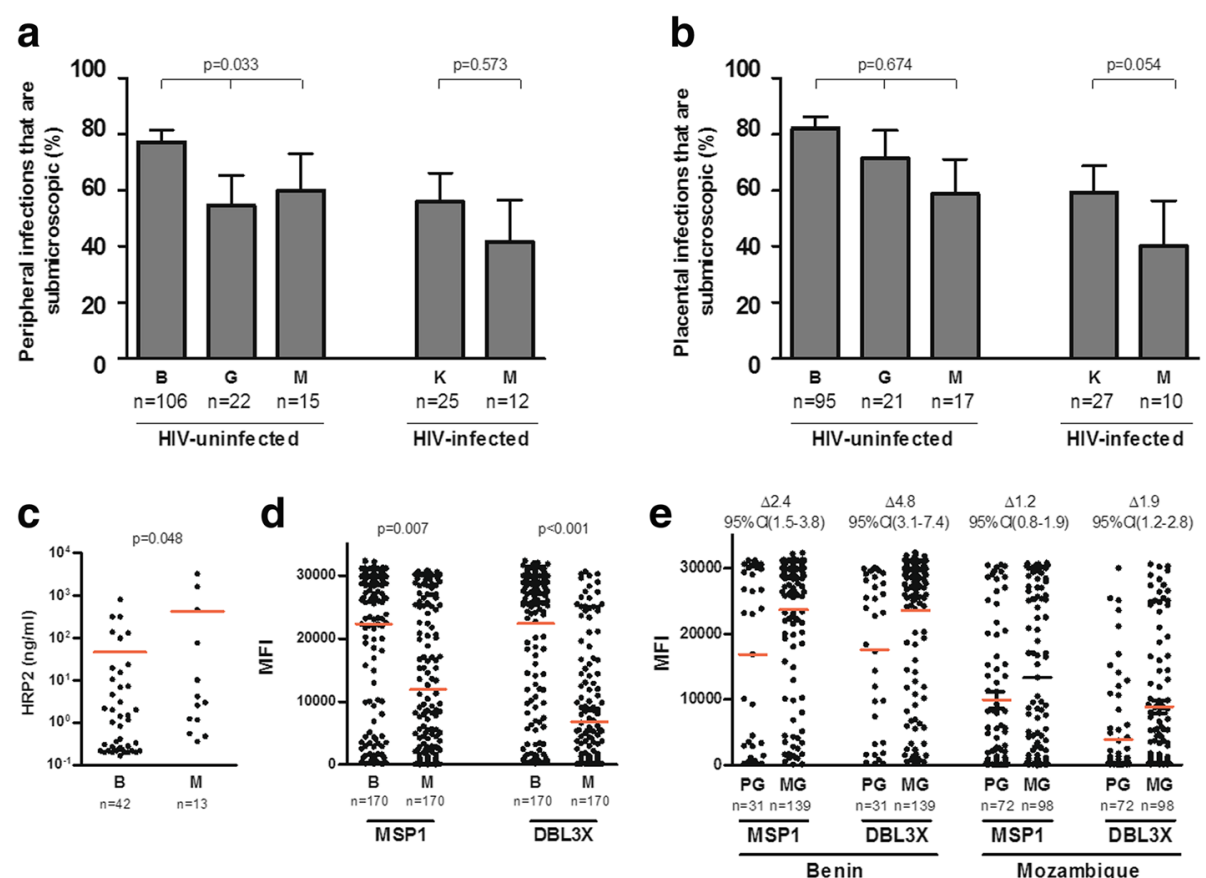

Fig. 3 Proportion of P. falciparum submicroscopic infections, PfHRP2 concentrations and antibody responses against $P$. falciparum antigens according to study area. Proportion of infections that are submicroscopic in peripheral (a) and placental (b) blood. $P$ value according to the multivariate analysis adjusted for type of IPTp drug, season, age, gravidity, gestational age, anaemia, literacy, RPR result and MUAC at recruitment, as well as CD4 + T cell count in the case of HIV-infected women. T bars represent standard errors. B Benin, G Gabon, M Mozambique, K Kenya. c PfHRP2 concentrations, indicative of overall parasite biomass, among women with a peripheral qPCR-detected infection in Mozambique $(M ; n=13)$ and Benin $(B ; n=42)$. $\mathbf{d}$ Levels of lgG antibodies against MSP1 and DBL3X in Beninese $(n=170)$ and Mozambican $(n=170)$ women. e Levels of IgG antibodies against MSP1 and DBL3X by parity (primigravidae $(P G)$ versus multigravidae $(M G)$ ), with the proportional increase $(\Delta)$ of the antibody levels plus $95 \%$ confidence interval in multigravidae versus primigravidae women. $P$ values obtained from regression analysis adjusted for type of IPTP drug, season, gravidity, gestational age, anaemia, literacy, RPR result and MUAC at recruitment, as well as CD4 + T cell count in the case of HIV-infected women. MFI mean fluorescence intensity

link between such a host defence strategy and malaria disease has been obscured by the difficulties of quantifying the tolerance phenotype. We sought to reduce the complexity of tolerance to a single metric based on the relationship between parasite densities and health outcomes (haemoglobin levels and birthweight) [8], with a flat slope indicative of tolerance to infection. To assess for variations in the levels of resistance to $P$. falciparum among women from the different endemic areas, we used the proportion of submicroscopic infections at delivery and IgG levels against parasite antigens as an indicator of the ability of pregnant women to restrict parasite growth [1]. Here, we have demonstrated that the lowest levels of immune resistance and tolerance observed among HIV-uninfected Mozambican women, compared to women from Benin, were accompanied by the largest adverse impacts of $P$. falciparum infections. Thus, the reduced severity of infections observed in pregnant women from high endemic regions [1] may not be mediated entirely by an adaptive immune response to the parasite, but also by a tolerance to P. falciparum, as quantified by the slope of the relationship between parasite densities and haemoglobin levels [8]. Taken together, these results provide evidence that pregnant women develop exposure-dependent mechanisms to minimise malaria pathology which, in concert with immune resistance, can reduce the adverse impact of $P$. falciparum infections.

Variation in resistance to $P$. falciparum among HIVuninfected women from three sub-Saharan African countries was suggested by different proportions of submicroscopic infections. Prevalence of qPCR-detected $P$. falciparum infections at delivery ranged from $41 \%$ in Benin to $11 \%$ in Gabon and $6 \%$ in Mozambique. Pregnant women from Benin had the highest proportion of submicroscopic infections at delivery, suggesting an increased capacity to maintain infections at densities bellow the detection limit of microscopy. These observations are in contrast with trends reported showing a significantly higher percentage of infections detected by microscopy in the general population residing in areas of high compared to those in low transmission areas [30]. These discrepancies may suggest a special dynamic and progression of $P$. falciparum infection during pregnancy compared to 


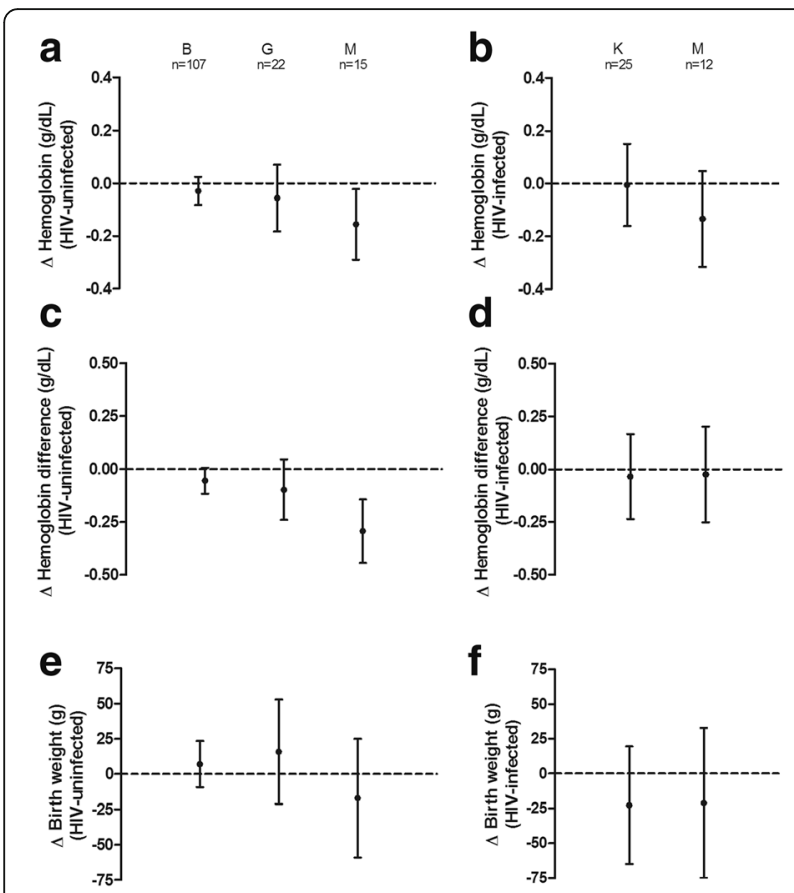

Fig. 4 Relationship of qPCR-detected parasite densities in peripheral blood with pregnancy outcomes. The dot and T bar represent the effect and 95\% confidence interval on haemoglobin levels at delivery $(\mathbf{a}, \mathbf{b})$, the difference of haemoglobin levels from recruitment to delivery (c, d), or birth weight (e, f) due to a two-fold increase in the parasitemia levels in its natural scale. Modification of the associations by study area ( $B$ Benin, $G$ Gabon, K Kenya, M Mozambique) was determined through the inclusion of an interaction term in the regression models, and combination of the coefficients plus the interaction and the standard error was estimated by the delta method. Analyses were adjusted for type of IPTp drug, season, age, gravidity, gestational age, anaemia, literacy, RPR result and MUAC at recruitment, as well as CD4 + T cell count in the case of HIV-infected women $(\mathbf{b}, \mathbf{d}, \mathbf{f})$

infections in non-pregnant hosts. In line with this, PfHPR2 levels in plasma, indicative of total parasite biomass [26], were lower among Beninese than Mozambican women, whereas levels of anti-parasite antibodies, as well as the increase with parity of IgGs against DBL3X from VAR2CSA, were higher in women from Benin than from Mozambique. Overall, these data supports the notion that the acquisition of antimalarial immunity after exposure to $P$. falciparum parasites can increase the capacity to resist P. falciparum growth during subsequent infections in pregnancy.

Variations in tolerance to $P$. falciparum were also observed in pregnant women living under contrasting levels of malaria transmission, as indicated by differences in the relationship between haemoglobin levels and increasing parasite burden. In this study, haemoglobin levels at delivery decreased as parasite densities increased in HIV-uninfected women from the lowest transmission setting in Mozambique. By contrast, the haemoglobin level in HIV-uninfected Beninese and
Gabonese women were not affected by parasite density, indicating a better tolerance to the infection [7]. Parasite factors such as the level of resistance to SP, as well as the protective effect of IPTp with SP against adverse birth outcomes that are related to curable sexually transmitted and reproductive tract infections [31, 32], may affect the clinical impact of infections among pregnant women who received antimalarials as IPTp. However, no relationship was observed between the level of molecular markers of SP resistance in the parasite population based on the frequencies of dihydropteroate synthase (Pfdhps) K540E mutation [33, 34] previously reported (>90\% Pfdhps-K540E in Kenya [33] but $<50 \%$ in Mozambique [35], Gabon [36] and Benin [37]) and the outcomes of the study. Moreover, the efficacy of IPTp with SP to clear peripheral parasites and prevent new infections during pregnancy has been suggested to be compromised only in areas with $>90 \%$ prevalence of Pfdhps K540E mutation [33]. Taken together, these results suggest that immunoregulatory responses that reduce pathogenic inflammation and potentially the risk of anaemia [38] may be developed by pregnant women exposed to $P$. falciparum, as has been suggested for children [39-41].

In absence of HIV infection, the adverse clinical impact of $P$. falciparum infections was the highest in pregnant women from the low transmission site in Mozambique, who had the lowest levels of immune resistance and tolerance to $P$. falciparum. This adverse impact was observed for microscopic $P$. falciparum infections, which were associated with reductions in maternal haemoglobin levels at delivery as well as with increased drops in haemoglobin levels from recruitment to delivery, but not with birthweight. Moreover, placental past/chronic infections among Mozambican women were associated with an increased risk of preterm births, in accordance with previous studies showing a larger impact of infections during pregnancy compared to infections detected only at delivery [42-44]. Such an adverse effect of placental past/chronic infections on the birthweight of newborns was not observed among Beninese or Gabonese women. P. falciparum microscopic infection in Gabonese women was also associated with an increased drop in haemoglobin levels from recruitment to delivery, although of a lower magnitude than the drop observed in Mozambican women. These data suggest that the malaria-related adverse impact in the health of pregnant women is higher in Gabon than in Benin, but lower than in Mozambique. Such an intermediate severity of the infections in Gabonese women might be explained by the development of tolerance to $P$. falciparum (as suggested by the lack of an association between haemoglobin levels with increasing parasite densities) rather than by immune resistance to the infection (as 
indicated by the similar carriage of submicroscopic infections in Gabonese and Mozambican women). Overall, this evidence suggests that resistance and tolerance to malaria can be acquired after exposure to $P$. falciparum parasites in areas of high transmission, and can reduce the detrimental consequences of $P$. falciparum infections. Importantly, HIV-infected pregnant women from Kenya and Mozambique did not show any evidence of varying levels of resistance or tolerance, suggesting that the ability to limit the adverse impact of $P$. falciparum infection may be reduced when the immune system is suppressed by the viral infection [45].

This study has some limitations. First, $P$. falciparum infection at delivery in women who received IPTp most likely reflect a recently acquired infection. Thus, this study may under-estimate the adverse impact of $P$. falciparum infections during pregnancy, as compared to recent reports showing that submicroscopic $P$. falciparum infection at inclusion (16.5 weeks) increases the risk of low birth weight for primigravid and premature delivery for multigravid pregnant Beninese women [6]. Second, other factors apart from anti-parasite immunity may contribute to the carriage of submicroscopic infections, such as the stage of the infection, the chronicity of infections, which is most common among primigravid women without immunity to placental parasites [46], and the existence of suppressive levels of antimalarial drugs. Third, site-specific differences in the extent of healthcare provided and economic development, as well as other factors than can affect pregnancy outcomes (i.e. haemoglobinopathies), might contribute to variations in the clinical impact of infections among pregnant women from different countries. However, the impact of these differences was minimised by the fact that this study was performed in the context of a clinical trial following standard procedures $[19,20]$ and that the analysis was adjusted by potential confounders. Fourth, the immunological analyses were conducted only in a subset of plasma samples available from the women included in the study, which were similar in covariates with the rest of the women. Fifth, analyses were performed by group, rather than at the individual level, assuming uniform exposure and risk among all individuals at each site. Finally, the small prevalence of $P$. falciparum infection among HIV-positive women who were receiving cotrimoxazole during pregnancy [20] may have reduced the power to detect variations in resistance and tolerance to malaria.

\section{Conclusions}

This study shows that pregnant women may reduce the deleterious impact of $P$. falciparum infection through two conceptually different types of defence against malaria, namely, one that targets the parasite (resistance), and the other that prevents the damage induced by the infection (tolerance) [7, 8]. Down-regulation of malariainduced inflammation may mediate this tolerance phenotype, although other mechanisms that increase the resilience to tissue injuries, such as metabolic adaptation to tissue repair and detoxification of pathogen byproducts [11, 47], might still be involved. Making the distinction between resistance mechanisms that restrict parasite multiplication from those that minimise the harm caused by the infection $[7,8]$ is important to understand how reductions in transmission intensity can affect host defences and, therefore, the clinical presentation of $P$. falciparum infections, as well as for the design of new host-directed therapies $[9,48,49]$.

\section{Additional file}

Additional file 1: Supplementary Tables and Figures. (DOCX 279 kb)

\section{Abbreviations}

DBL3X: Duffy-Binding Like 3 X; HRP2: Histidine-Rich Protein2; IPTp: intermittent preventive treatment during pregnancy; MQ: mefloquine; MSP1: Merozoite Surfce Protein 1; P. falciparum: Plasmodium falciparum; QPCR: quantitative polymerase chain-reaction; SP: sulfadoxine-pyrimethamine

\section{Acknowledgments}

We are grateful to the women from Benin, Gabon, Kenya and Mozambique participating in the study; the staff of the Hospitals, clinical officers, field supervisors and data manager; Dr Chetan Chitnis for his contribution with the recombinant proteins used in the Luminex-based assays; Laura Puyol, Lázaro Mussacate, Nelito Ernesto José, Ana Rosa Manhiça, Augusto Nhabomba and Chenjerai S. Jairoce for their contribution to the collection and analysis of samples and Jaume Ordi for placental histologies. The findings and conclusions of this report are those of the authors and do not necessarily represent the views of the $C D C$

\section{Funding}

This work was supported by Instituto de Salud Carlos III (PI13/01478) 2013 call for the Strategic Action on Health 2013-2016 co-funded by the Fondo Europeo de Desarrollo Regional (FEDER), CES10/021-I3SNS and 2014SGR263 from the Departament d'Universitats i Recerca de la Generalitat de Catalunya (AGAUR) to AM, EDCTP, Malaria in Pregnancy (MiP) Consortium, and Malaria Eradication Scientific Alliance (MESA). ISGlobal is a member of the CERCA Programme, Generalitat de Catalunya. The Manhiça Health Research Centre receives core support from the Spanish Agency for International Cooperation and Development. The MiP Consortium and MESA are funded through a grant from the Bill \& Melinda Gates Foundation to the Liverpool School of Tropical Medicine and the Barcelona Institute of Global Health (ISGlobal), respectively.

\section{Availability of data and material}

The datasets used and/or analysed during the current study are available from the corresponding author on reasonable request.

\section{Authors' contributions}

AM: Conception and design, acquisition of data, analysis and interpretation of data, drafting and revising the article. NDN, EM: acquisition of data, analysis and interpretation of data, drafting and revising the article. LQ: analysis and interpretation of data. PC, AMF, AJ, RG, SK, ES, MR, AV, SM, PO, $M R$, JJA, AN, AC, VB, PGK, GMN, MD, EM, MC, CM: acquisition of data and interpretation of data. All authors read and approved the final manuscript. 


\section{Consent for publication}

Not applicable.

\section{Ethics approval and consent to participate}

The study was approved by the Ethics Committees from the Hospital Clínic of Barcelona (Spain), the Comité Consultatif de Déontologie et d'Éthique from the Institut de Recherche pour le Développement (France), the Centers for Disease Control and Prevention (USA), and National Ethics Review Committees from each malaria endemic country. Study participants signed a written informed consent form prior to enrolment.

\section{Publisher's Note}

Springer Nature remains neutral with regard to jurisdictional claims in published maps and institutional affiliations.

\section{Author details}

IInstitut de Recherche pour le Développement (IRD), Paris, France. ${ }^{2}$ COMUE Sorbonne Paris Cité, Faculté de Pharmacie, Paris, France. ${ }^{3}$ Faculté des Sciences de la Santé (FSS), Université d'Aboméy Calavi, Cotonou, Benin. ${ }^{4}$ Ifakara Health Institute (IHI), Bagamoyo Research and Training Centre (BRTC), Bagamoyo, Tanzania. ${ }^{5}$ ISGlobal, Barcelona Ctr. Int. Health Res. (CRESIB), Hospital Clínic - Universitat de Barcelona, Barcelona, Spain. ${ }^{6}$ Centro de Investigação em Saúde da Manhiça (CISM), Maputo, Mozambique. ${ }^{7}$ Universidade Eduardo Mondlane, Maputo, Mozambique. ${ }^{8}$ Kenya Medical Research Institute (KEMRI)/Centre for Global Health Research, Kisumu, Kenya ${ }^{9}$ Graduate Program in Areas of Basic and Applied Biology, Universidade do Porto, Porto, Portugal. ${ }^{10} \mathrm{CIBER}$ Epidemiología y Salud Pública (CIBERESP), Madrid, Spain. ${ }^{11}$ Institute of Tropical Medicine, University of Tübingen, Tübingen, Germany. ${ }^{12}$ Department of Medicine I, Division of Infectious Diseases and Tropical Medicine, Medical University of Vienna, Vienna, Austria. ${ }^{13}$ Centre de Recherches Médicales de Lambaréné (CERMEL), Lambaréné, Gabon. ${ }^{14}$ Malaria Branch, Division of Parasitic Diseases and Malaria, Center for Global Health, Centers for Disease Control and Prevention, Atlanta, Georgia, USA.

Received: 22 February 2017 Accepted: 15 June 2017

Published online: 17 July 2017

\section{References}

1. Mayor A, Bardaji A, Macete E, Nhampossa T, Fonseca AM, Gonzalez R, Maculuve S, Cistero P, Ruperez M, Campo J, et al. Changing trends in P. falciparum burden, immunity, and disease in pregnancy. N Engl J Med. 2015:373(17):1607-17.

2. Nosten F, Rogerson SJ, Beeson JG, McGready R, Mutabingwa TK, Brabin B. Malaria in pregnancy and the endemicity spectrum: what can we learn? Trends Parasitol. 2004;20(9):425-32.

3. Ataide R, Mayor A, Rogerson SJ. Malaria, primigravidae, and antibodies: knowledge gained and future perspectives. Trends Parasitol. 2014;30(2):85-94.

4. Mockenhaupt FP, Rong B, Till H, Eggelte TA, Beck S, Gyasi-Sarpong C, Thompson WN, Bienzle U. Submicroscopic Plasmodium falciparum infections in pregnancy in Ghana. Trop Med Int Health. 2000;5(3):167-73.

5. Walker-Abbey A, Djokam RR, Eno A, Leke RF, Titanji VP, Fogako J, Sama G, Thuita LH, Beardslee E, Snounou G, et al. Malaria in pregnant Cameroonian women: the effect of age and gravidity on submicroscopic and mixedspecies infections and multiple parasite genotypes. Am J Trop Med Hyg. 2005:72(3):229-35.

6. Cottrell G, Moussiliou A, Luty AJ, Cot M, Fievet N, Massougbodji A, Deloron P. Tuikue NN. Submicroscopic Plasmodium falciparum infections are associated with maternal anemia, premature births and low birthweight Clin Infect Dis. 2015:60(10):1481-8.

7. Medzhitov R, Schneider DS, Soares MP. Disease tolerance as a defense strategy. Science. 2012;335(6071):936-41.

8. Raberg L, Sim D, Read AF. Disentangling genetic variation for resistance and tolerance to infectious diseases in animals. Science. 2007;318(5851):812-4.

9. Zumla A, Rao M, Wallis RS, Kaufmann SH, Rustomjee R, Mwaba P, Vilaplana C, Yeboah-Manu D, Chakaya J, Ippolito G, et al. Host-directed therapies for infectious diseases: current status, recent progress, and future prospects. Lancet Infect Dis. 2016;16(4):e47-63.

10. Raberg L. How to live with the enemy: understanding tolerance to parasites. PLoS Biol. 2014;12(11), e1001989.
11. Galatas B, Bassat Q, Mayor A. Malaria parasites in the asymptomatic: looking for the hay in the haystack. Trends Parasitol. 2016:32(4):296-308.

12. Collins WE, Jeffery GM. A retrospective examination of sporozoite- and trophozoite-induced infections with Plasmodium falciparum: development of parasitologic and clinical immunity during primary infection. Am J Trop Med Hyg. 1999:61(1 Suppl):4-19.

13. Gupta S, Snow RW, Donnelly CA, Marsh K, Newbold C. Immunity to noncerebral severe malaria is acquired after one or two infections. Nat Med. 1999:5(3):340-3.

14. Rogier C, Commenges D, Trape JF. Evidence for an age-dependent pyrogenic threshold of Plasmodium falciparum parasitemia in highly endemic populations. Am J Trop Med Hyg. 1996;54(6):613-9.

15. Smith T, Killeen $G$, Lengeler $C$, Tanner M. Relationships between the outcome of Plasmodium falciparum infection and the intensity of transmission in Africa. Am J Trop Med Hyg. 2004;71(2 Suppl):80-6.

16. Petersen E, Hogh B, Marbiah NT, David K, Hanson AP. Development of immunity against Plasmodium falciparum malaria: clinical and parasitologic immunity cannot be separated. J Infect Dis. 1991;164(5):949-53.

17. Bardaji A, Sigauque B, Romagosa C, Bruni L, Sanz S, Mabunda S, Mandomando I, Aponte J, Sevene E, Alonso PL, et al. Clinical malaria in African pregnant women. Malar J. 2008:7(1):27.

18. O'Neil-Dunne I, Achur RN, Agbor-Enoh ST, Valiyaveettil M, Naik RS, Ockenhouse CF, Zhou A, Megnekou R, Leke R, Taylor DW, et al. Graviditydependent production of antibodies that inhibit binding of Plasmodium falciparum-infected erythrocytes to placental chondroitin sulfate proteoglycan during pregnancy. Infect Immun. 2001;69(12):7487-92.

19. Gonzalez R, Mombo-Ngoma G, Ouedraogo S, Kakolwa MA, Abdulla S, Accrombessi M, Aponte JJ, Akerey-Diop D, Basra A, Briand V, et al. Intermittent preventive treatment of malaria in pregnancy with mefloquine in HIV-negative women: a multicentre randomized controlled trial. PLoS Med. 2014;11(9), e1001733.

20. Gonzalez R, Desai M, Macete E, Ouma P, Kakolwa MA, Abdulla S, Aponte JJ, Bulo $\mathrm{H}$, Kabanywanyi AM, Katana A, et al. Intermittent preventive treatment of malaria in pregnancy with mefloquine in HIV-infected women receiving cotrimoxazole prophylaxis: a multicenter randomized placebo-controlled trial. PLoS Med. 2014;11(9), e1001735

21. Ballard JL, Khoury JC, Wedig K, Wang L, Eilers-Walsman BL, Lipp R. New Ballard Score, expanded to include extremely premature infants. J Pediatr. 1991;119(3):417-23.

22. Greenwood AM, Armstrong JR, Byass P, Snow RW, Greenwood BM. Malaria chemoprophylaxis, birth weight and child survival. Trans R Soc Trop Med Hyg. 1992;86(5):483-5

23. Ordi J, Ismail MR, Ventura PJ, Kahigwa E, Hirt R, Cardesa A, Alonso PL, Menendez C. Massive chronic intervillositis of the placenta associated with malaria infection. Am J Surg Pathol. 1998;22(8):1006-11.

24. Joanny F, Lohr SJ, Engleitner T, Lell B, Mordmuller B. Limit of blank and limit of detection of Plasmodium falciparum thick blood smear microscopy in a routine setting in Central Africa. Malar J. 2014;13:234.

25. Taylor SM, Mayor A, Mombo-Ngoma G, Kenguele HM, Ouedraogo S, Ndam NT, Mkali H, Mwangoka G, Valecha N, Singh JP, et al. A quality control program within a clinical trial Consortium for PCR protocols to detect Plasmodium species. J Clin Microbiol. 2014:52(6):2144-9.

26. Dondorp AM, Desakorn V, Pongtavornpinyo W, Sahassananda D, Silamut K, Chotivanich K, Newton PN, Pitisuttithum P, Smithyman AM, White NJ, et al. Estimation of the total parasite biomass in acute falciparum malaria from plasma PfHRP2. PLoS Med. 2005;2(8), e204.

27. Mayor A, Moro L, Aguilar R, Bardaji A, Cistero P, Serra-Casas E, Sigauque B, Alonso PL, Ordi J, Menendez C. How hidden can malaria be in pregnant women? Diagnosis by microscopy, placental histology, polymerase chain reaction and detection of histidine-rich protein 2 in plasma. Clin Infect Dis. 2012;54(11):1561-8.

28. Mayor A, Serra-Casas E, Bardaji A, Sanz S, Puyol L, Cistero P, Sigauque B, Mandomando I, Aponte JJ, Alonso PL, et al. Sub-microscopic infections and long-term recrudescence of Plasmodium falciparum in Mozambican pregnant women. Malar J. 2009:8:9.

29. Sinton JA. Immunity or tolerance in malarial infections: (section of comparative medicine). Proc R Soc Med. 1938;31(11):1298-302.

30. Okell LC, Ghani AC, Lyons E, Drakeley CJ. Submicroscopic infection in Plasmodium falciparum-endemic populations: a systematic review and meta-analysis. J Infect Dis. 2009;200(10):1509-17.

31. Chico RM, Cano J, Ariti C, Collier TJ, Chandramohan D, Roper C, Greenwood B. Influence of malaria transmission intensity and the $581 \mathrm{G}$ mutation on the 
efficacy of intermittent preventive treatment in pregnancy: systematic review and meta-analysis. Trop Med Int Health. 2015;20(12):1621-33.

32. Chico RM, Chaponda EB, Ariti C, Chandramohan D. Sulfadoxinepyrimethamine exhibits dose-response protection against adverse birth outcomes related to malaria and sexually transmitted and reproductive tract infections. Clin Infect Dis. 2017:64(8):1043-51.

33. Desai M, Gutman J, Taylor SM, Wiegand RE, Khairallah C, Kayentao K, Ouma $\mathrm{P}$, Coulibaly SO, Kalilani L, Mace KE, et al. Impact of sulfadoxinepyrimethamine resistance on effectiveness of intermittent preventive therapy for malaria in pregnancy at clearing infections and preventing low birth weight. Clin Infect Dis. 2016;62(3):323-33.

34. Naidoo I, Roper C. Drug resistance maps to guide intermittent preventive treatment of malaria in African infants. Parasitology. 2011;138(12): 1469-79.

35. Menendez C, Serra-Casas E, Scahill MD, Sanz S, Nhabomba A, Bardaji A, Sigauque B, Cistero P, Mandomando I, Dobano C, et al. HIV and placental infection modulate the appearance of drug-resistant Plasmodium falciparum in pregnant women who receive intermittent preventive treatment. Clin Infect Dis. 2011:52(1):41-8.

36. Mombo-Ngoma G, Oyakhirome S, Ord R, Gabor JJ, Greutelaers KC, Profanter K, Greutelaers B, Kurth F, Lell B, Kun JF, et al. High prevalence of dhfr triple mutant and correlation with high rates of sulphadoxinepyrimethamine treatment failures in vivo in Gabonese children. Malar J. 2011;10:123.

37. Ogouyemi-Hounto A, Ndam NT, Fadegnon G, Azagnandji C, Bello M, Moussiliou A, Chippaux JP, Kinde Gazard D, Massougbodji A. Low prevalence of the molecular markers of Plasmodium falciparum resistance to chloroquine and sulphadoxine/pyrimethamine in asymptomatic children in Northern Benin. Malar J. 2013;12:413.

38. Artavanis-Tsakonas K, Tongren JE, Riley EM. The war between the malaria parasite and the immune system: immunity, immunoregulation and immunopathology. Clin Exp Immunol. 2003;133(2):145-52.

39. Portugal S, Moebius J, Skinner J, Doumbo S, Doumtabe D, Kone Y, Dia S, Kanakabandi K, Sturdevant DE, Virtaneva K, et al. Exposure-dependent control of malaria-induced inflammation in children. PLoS Pathog. 2014; 10(4), e1004079.

40. Jagannathan P, Eccles-James I, Bowen K, Nankya F, Auma A, Wamala S, Ebusu C, Muhindo MK, Arinaitwe E, Briggs J, et al. IFNgamma/IL-10 coproducing cells dominate the CD4 response to malaria in highly exposed children. PLoS Pathog. 2014;10(1), e1003864.

41. Tran TM, Jones MB, Ongoiba A, Bijker EM, Schats R, Venepally P, Skinner J, Doumbo S, Quinten E, Visser LG, et al. Transcriptomic evidence for modulation of host inflammatory responses during febrile Plasmodium falciparum malaria. Sci Rep. 2016;6:31291.

42. Cottrell G, Mary JY, Barro D, Cot M. The importance of the period of malarial infection during pregnancy on birth weight in tropical Africa. Am J Trop Med Hyg. 2007;76(5):849-54

43. Huynh BT, Fievet N, Gbaguidi G, Dechavanne S, Borgella S, Guezo-Mevo B, Massougbodji A, Ndam NT, Deloron P, Cot M. Influence of the timing of malaria infection during pregnancy on birth weight and on maternal anemia in Benin. Am J Trop Med Hyg. 2011;85(2):214-20.

44. Valea I, Tinto H, Drabo MK, Huybregts L, Sorgho H, Ouedraogo JB, Guiguemde RT, van Geertruyden JP, Kolsteren P, D'Alessandro U, et al. An analysis of timing and frequency of malaria infection during pregnancy in relation to the risk of low birth weight, anaemia and perinatal mortality in Burkina Faso. Malar J. 2012;11:71.

45. Gonzalez R, Ataide R, Naniche D, Menendez C, Mayor A. HIV and malaria interactions: where do we stand? Expert Rev Anti Infect Ther. 2012;10(2): 153-65.

46. Brabin BJ, Romagosa C, Abdelgalil S, Menendez C, Verhoeff FH, McGready R, Fletcher KA, Owens S, D'Alessandro U, Nosten F, et al. The sick placenta-the role of malaria. Placenta. 2004;25(5):359-78.

47. Liu M, Hassana S, Stiles JK. Heme-mediated apoptosis and fusion damage in BeWo trophoblast cells. Sci Rep. 2016;6:36193.

48. Higgins SJ, Purcell LA, Silver KL, Tran V, Crowley V, Hawkes M, Conroy AL, Opoka RO, Hay JG, Quaggin SE, et al. Dysregulation of angiopoietin-1 plays a mechanistic role in the pathogenesis of cerebral malaria. Sci Transl Med. 2016:8(358):358ra128.

49. Higgins SJ, Kain KC, Liles WC. Immunopathogenesis of falciparum malaria: implications for adjunctive therapy in the management of severe and cerebral malaria. Expert Rev Anti Infect Ther. 2011;9(9):803-19.

\section{Submit your next manuscript to BioMed Central and we will help you at every step:}

- We accept pre-submission inquiries

- Our selector tool helps you to find the most relevant journal

- We provide round the clock customer support

- Convenient online submission

- Thorough peer review

- Inclusion in PubMed and all major indexing services

- Maximum visibility for your research

Submit your manuscript at www.biomedcentral.com/submit
) Biomed Central 